\title{
Prediction of corrugation in rails using a non-stationary wheel-rail contact model
}

\author{
Luis Baeza ${ }^{\mathrm{a}, *}$, Paloma Vila ${ }^{\mathrm{a}}$, Alejandro Roda ${ }^{\mathrm{a}}$, Juan Fayos ${ }^{\mathrm{b}}$ \\ a Dpto. Ingeniería Mecánica y Materiales, Universidad Politécnica de Valencia, Camino de Vera s/n, 46022 Valencia, Spain \\ b Instituto de Biomecánica de Valencia, Universidad Politécnica de Valencia, Spain
}

\section{A R T I C L E I N F O}

\section{Article history:}

Accepted 14 January 2008

Available online 19 May 2008

\section{Keywords:}

Corrugation

Non-steady state rolling contact

\begin{abstract}
A B S T R A C T
Most of the models used for simulating the conditions existing in the wheel-rail contact are based on stationary theories. In such theories, the parameters associated with the wheel-rail contact are independent on the conditions applied on it previously. This supposition is a simplification of the real phenomenon, whose validity lies in the rapid convergence of the contact parameters to their stationary values. However, the conditions simulated by means of non-stationary theories may differ from those obtained by using stationary theories when external conditions vary rapidly. Certain types of rail corrugation may be related to high-frequency normal or tangential forces transmitted through the contact, which may determine the effect of the temporal history on the contact parameters, and consequently on the rail wear. In order to investigate the influence of the contact process on the results of models of corrugation calculation, a methodology for estimating the rail wear depth due to a wheel running on a stretch of rail is developed. The method implements an improved contact model where non-stationary hypotheses and an exact elastic model are taken into account. The results show the influence of the more realistic hypotheses adopted in the proposed method.
\end{abstract}

(C) 2008 Luis Baeza. Published by Elsevier B.V. All rights reserved.

\section{Introduction}

Rail corrugation has been a problem for railway industry as well as a subject of investigation by engineers all over the world for more than a century. Rolling noise, vibration, deterioration of vehicle and track components, along with discomfort to passengers are its principal negative effects. Nevertheless, the present operating conditions of railway vehicles, with higher speeds in passenger services and greater axle loads in freight trains, together with a general increase in railway traffic, make rail corrugation an even more serious problem. There is thus an incentive to study the causes and development of this phenomenon in order to prevent or minimise its effects.

This defect appears in all types of tracks, due to the continual passage of wheelsets, as a periodic undulation with a certain wavelength on the running surface of the rail. Grassie and Kalousek initially classified rail corrugation into six types [1]. Later, finding the typology to be even more extensive, they proposed classifying the phenomenon according to a wavelength-fixing mechanism related to resonance either from wheelsets, rails or the coupled

\footnotetext{
* Corresponding author.

E-mail address: lbaeza@mcm.upv.es (L. Baeza).
}

vehicle-track system, and a damage mechanism such as plastic deformation or wear [2]. These authors suggested that wear is the damage mechanism responsible for most types of corrugation.

Since the problem is complex, mathematical models to simulate the beginning and evolution of corrugation are an essential tool for its analysis. Some of the existing models combine three mechanical models: a vehicle-track dynamic interaction model, a rolling contact model and a wear model [3-6]. The irregularities initially present on the rail running surface cause variations in the wheelrail contact forces, in creepage and in the size of the contact patch. The input data of the wear model is composed of the results given by the vehicle-track dynamic interaction models and the contact model. This approach was developed from the specially designed tools for classic railway dynamics, such as the FASTSIM algorithm [7], to calculate contact parameters.

FASTSIM provides excellent results in determining the relationship between creepage and contact-transmitted forces, also for calculating the stick and slip zones in the contact patch. It also has the advantage of a low computational cost since it employs a simplified elastic model. The elastic model considers that the displacements associated with the elastic deformations at a point in the contact patch are a linear function of the traction forces applied at that point (Simplified Theory), also known as Winkler's model or Elastic layer. The effect of this simplified assumption was corrected 
so that FASTSIM provides the same results than Kalker's Linear Theory at low external force or creepage. However, the local slip calculation precision may be affected by the elastic model hypothesis since this parameter has not been adjusted. This problem could influence the calculations because the local slip is the input data in wear models. The enhancing of the contact model can therefore be a way of improving methods for estimating rail wear.

An approach in this sense was presented by Li [8] and Jin et al. [9-11]. Li proposed a method for calculating wheel wear through non-Hertzian normal contact models. In Jin's studies, an improved normal contact model (non-Hertzian) was considered which permitted greater precision in determining the coupled dynamics between track and vehicle. The last method was implemented in rail wear calculations. Another work that followed this approach, but applied to wheelflats, was that of [12] who made use of the dynamic model proposed in [13].

The non-stationary contact process was investigated by Kalker [7] and Gross-Thebing and Knothe [14-18]. Gross-Thebing et al. studied the influence of the external tangential force variation on creep coefficients due to non-stationary contact process. Kalker developed a model to calculate the non-stationary contact process based on an Exact Theory (non-simplified relationships between displacements and tractions in the contact patch). Nevertheless, he concluded that its applicability to practical cases was inadequate due to the rapid convergence of the contact process to its stationary values. The non-stationary model has a much higher computational cost than FASTSIM or the Linear Theory, a disadvantage that prevented it from being used when the model was developed.

The applicability of a non-stationary contact model could however be related to the calculation of the contact parameters associated with a corrugated rail. Nielsen [19] thus developed a methodology to solve the tangential contact problem according to a bi-dimensional theory applied to the case of a corrugated rail. From its conclusions, the influence of the non-stationary process on wear results is determined when the forces involved in the contact vary rapidly.

The aim of the present work is to propose a method for estimating rail wear in three dimensions by using an improved contact model. The model input data are the contact geometry, the mechanical properties of the materials and the resulting forces transmitted between rail and wheel. The output gives the wear depth on the railhead. Regarding the models used for estimating wear, the contact model is based on an Exact Theory and considers the non-stationary wheel-rail contact process.

In the following section, the calculation model of the contact parameters is described. These parameters include the local slip used as input for the wear model implemented (Archard [20]). Section 3 establishes the procedure for the prediction of rail wear. In Section 4 , the developed calculation method is applied to cases that could be considered as real and the results are compared to those of FASTSIM. The parameters of the model used in the calculations are given in Table 1.

Table 1

Model parameters

\begin{tabular}{ll}
\hline Radius of the railhead curvature & $300 \mathrm{~mm}$ \\
Radius of the wheel profile & $409 \mathrm{~mm}$ \\
Wheel radius & $500 \mathrm{~mm}$ \\
Vehicle speed & $100 \mathrm{~km} / \mathrm{h}$ \\
Friction coefficient & 0.4 \\
Young's modulus & $2.1 \times 10^{11} \mathrm{~N} / \mathrm{m}^{2}$ \\
Poisson ratio & 0.3 \\
Normal force mean value & $100 \mathrm{kN}$ \\
Longitudinal force mean value & $20 \mathrm{kN}$ \\
$K_{\mathrm{w}} / \mathrm{H}$ ratio for Archard model & $3.40 \times 10^{-14} \mathrm{~m}^{2} / \mathrm{N}$ \\
\hline
\end{tabular}

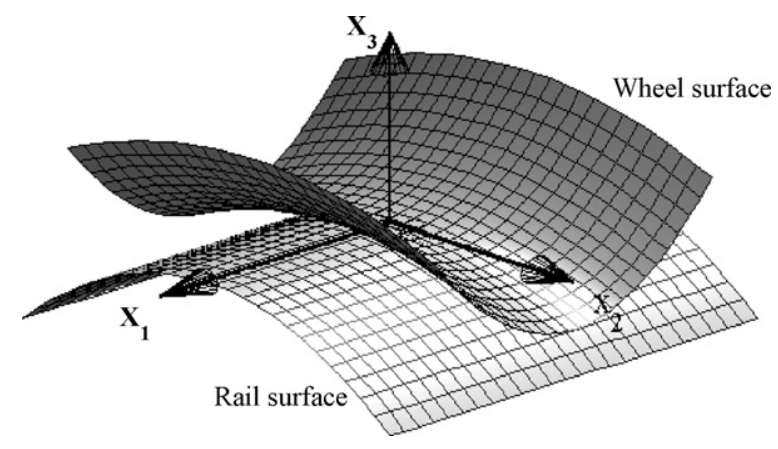

Fig. 1. Coordinate system.

\section{Contact model}

The method employed to calculate the contact parameters is based on the non-stationary model developed by Kalker [7]. With respect to the original model, a discretisation adapted to the contact area is used with the aim of enhancing the quality of the solution when variations occur in the normal contact forces. A wide description of the method can be found in [21].

The hypotheses associated with the non-conformal contact are considered, and it is also assumed that the normal (Hertzian) contact problem does not depend on the tangential contact problem. Its formulation is based on two wheel-rail contact models, one kinetic and one elastic. Both models will use a system of mobile coordinates $x_{1} x_{2} x_{3}$ (see Fig. 1) whose origin is situated at the centre of the contact patch; the $x_{1}$ axis defines the direction of the wheel movement and the contact plane corresponds with $x_{3}=0$.

To formulate the tangential contact problem, the following magnitudes are defined referring to the previously mentioned reference system

- The creepages $\xi_{1}$ and $\xi_{2}$, and spin $\xi_{\text {sp }}$, or the theoretical contact point velocities normalised in relation to the vehicle velocity $V$.

- The local slip s, or the relative velocity between the contact surfaces.

- The displacements associated with the elastic deformations $\mathbf{u}$.

- The tractions transmitted from rail to wheel through the contact patch p.

The relations between the magnitudes corresponding to the kinetic model are as follows:

$\mathbf{s}=V\left\{\begin{array}{l}\xi_{1}-x_{2} \xi_{\text {sp }} \\ \xi_{2}+x_{1} \xi_{\text {sp }}\end{array}\right\}+2 \frac{\partial \mathbf{u}}{\partial t}$

And those corresponding to the elastic model $(\tau=1,2)$ are

$u_{\tau}\left(x_{1}, x_{2}\right)=\sum_{\kappa=1}^{2} \iint_{\text {Contact area }} A_{\tau \kappa}\left(x_{1}, x_{2}, y_{1}, y_{2}\right) p_{\kappa}\left(y_{1}, y_{2}\right) \mathrm{d} y_{1} \mathrm{~d} y_{2}$

where $A_{\tau \kappa}$ are the elastic influence coefficients. The procedure requires a double discretisation of the problem. The first discretisation is done in the spatial domain and consists of dividing the contact area into a Paul and Hashemi mesh [22] of the type shown in Fig. 2. The local slip, the traction and the displacements associated with the elastic deformations are assumed to be constant inside each element and equal to the value they would acquire at the centre of the element. According to this simplified hypothesis, 


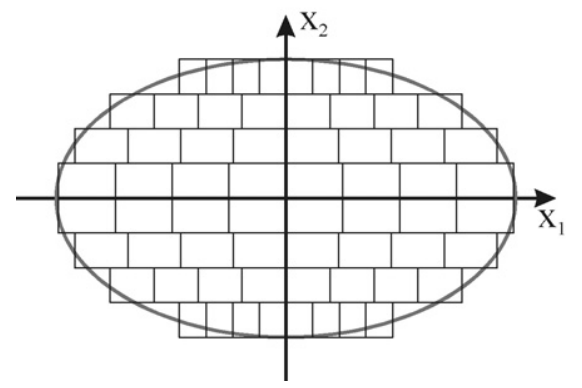

Fig. 2. Contact area discretisation according to Paul and Hashemi's model.

Eq. (1) for the $J$-th element would be

$\left\{\begin{array}{l}s_{1}^{J} \\ s_{2}^{J}\end{array}\right\}=V\left\{\begin{array}{l}\xi_{1}-x_{2}^{J} \xi_{\mathrm{sp}} \\ \xi_{2}+x_{1}^{J} \xi_{\mathrm{sp}}\end{array}\right\}+2 \frac{\partial \mathbf{u}^{J}}{\partial t}$

and from Eq. (2) we obtain

$u_{\tau}^{J}=\sum_{\kappa=1}^{2} \sum_{K=1}^{N} D_{\tau \kappa}^{J K} p_{\kappa}^{K} \quad \tau=1,2$

where the values $D_{\tau \kappa}^{J K}$ are calculated under the assumption that the bodies behave elastically as infinite half-spaces, being $N$ the number of elements.

The next discretisation of the problem is performed in the time domain and involves establishing an approximation by finite differences of the value of the time derivative in (3) according to

$\frac{\partial \mathbf{u}^{J}}{\partial t} \approx \frac{\mathbf{u}^{J}-\mathbf{u}_{o}^{J}}{\Delta t}$

To consider local slip and its relation to contact tractions, a regularisation model of Coulomb's law is considered, in which $\mu$ stands for the friction coefficient,

$p_{\tau}^{J} \approx-\frac{2}{\pi} \operatorname{atan}\left(\frac{\left\|\mathbf{s}^{J}\right\|}{\varepsilon}\right) \frac{\mu p_{3}^{J} s_{\tau}^{J}}{\left\|\mathbf{S}^{J}\right\|}$

where $\varepsilon$ is the regularisation parameter. Expression (6) converges exactly to Coulomb's law when $\varepsilon$ tends to zero (the model provides a good approach if $\varepsilon=10^{-5} \mathrm{~m} / \mathrm{s}$ ). The problem is formulated through local slip when (6) is substituted in (4), and (4) and (5) in (3). The solution to the tangential contact problem is obtained through the following system of equations

$$
\begin{aligned}
\left\{\begin{array}{l}
s_{1}^{J} \\
s_{2}^{J}
\end{array}\right\}= & V\left\{\begin{array}{l}
\xi_{1}-x_{2}^{J} \xi_{\mathrm{sp}} \\
\xi_{2}+x_{1}^{J} \xi_{\mathrm{sp}}
\end{array}\right\} \\
& -\frac{4 \mu}{\pi \Delta t} \sum_{\kappa=1}^{2} \sum_{K=1}^{N}\left(\frac{p_{3}^{K} \operatorname{atan}\left(\left\|\mathbf{s}^{K}\right\| / \varepsilon\right)}{\left\|\mathbf{s}^{K}\right\|}\left\{\begin{array}{l}
D_{1 \kappa}^{J K} s_{\kappa}^{K} \\
D_{2 \kappa}^{J K} s_{\kappa}^{K}
\end{array}\right\}\right)-\frac{2}{\Delta t} \mathbf{u}_{o}^{J}
\end{aligned}
$$

The nonlinear algebraic system associated with (7) is solved via Newton-Raphson through a previous estimation of the solution.

The results of the presented contact model yield contact parameter fields that cannot be calculated through models based on a stationary theory. Figs. 3 and 4 show examples of these cases. These calculations correspond to the longitudinal traction distribution in the contact area for an instant when one external force transmitted through the contact varies harmonically around its mean value. Fig. 3 considers the variation of the external normal force. We can observe that the maximal traction value $\mu p_{3}$ is reached in the leading edge of the contact patch, and consequently there is a slip area

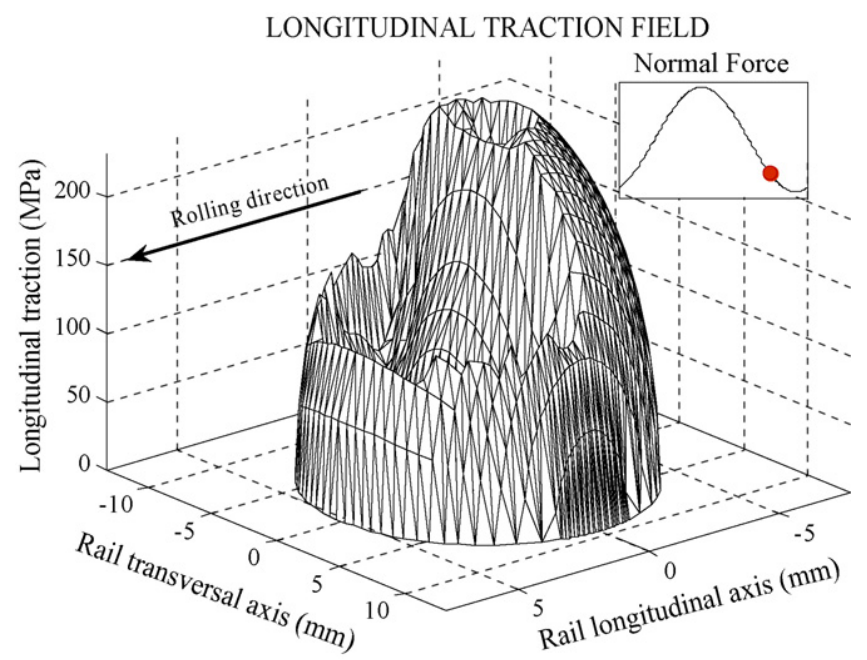

Fig. 3. Longitudinal traction distribution computed through a non-stationary contact model. Load case: longitudinal constant force and harmonic variation of the normal force. The normal force subplot shows which instant in cycle is represented. The complete cycle can be found in the Electronic Annex 1 in the online version of this article.

in this zone. On the other hand, the longitudinal force variation can be analysed in Fig. 4. As the precedent case, we can distinguish the slip area in the leading edge of the contact patch, but the direction of the traction and local slip is reverse. These aspects may influence the results of the wear calculation models based on the local slip.

\section{Wear calculation}

The proposed method for estimating the worn profile of the rail rolling surface aims to calculate the wear depth at the nodes of the regular mesh by which this surface is discretised. It is based on a combination of a wheel-rail contact model, described in Section 2, and a wear model. The Archard wear model is chosen for this study since it is the most frequently used in tribology for modelling sliding wear.

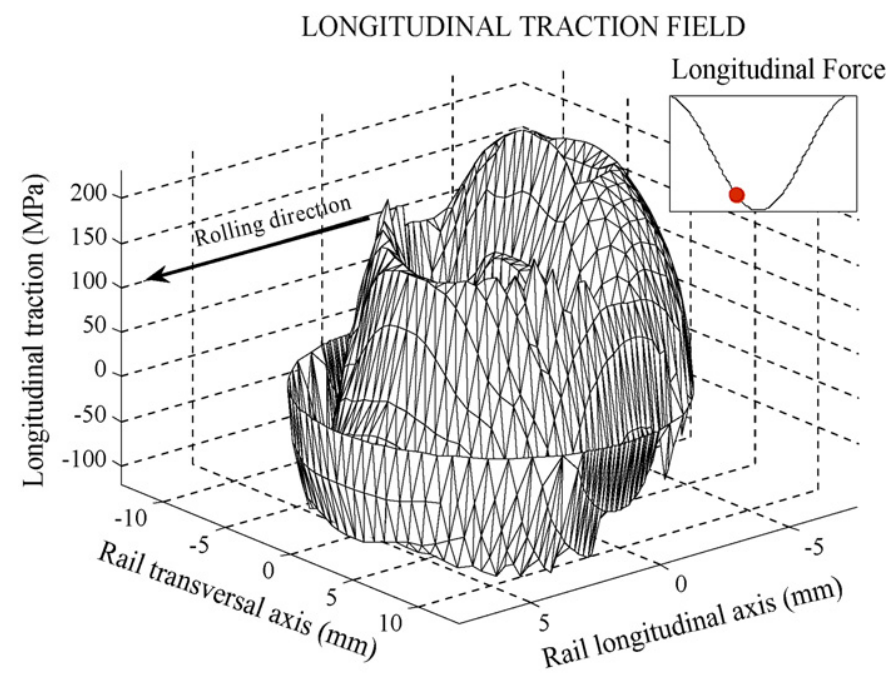

Fig. 4. Longitudinal traction distribution computed through a non-stationary contact model. Load case: normal constant force and harmonic variation of the longitudinal force. The longitudinal force subplot shows which instant in cycle is represented. The complete cycle can be found in the Electronic Annex 2 in the online version of this article. 
Archard's model establishes that the wear rate $\dot{w}(\mathrm{~m} / \mathrm{s})$ is directly proportional to the normal tension applied to the surface $p_{3}$ and to the local slip modulus $\|\mathbf{S}\|$, and inversely proportional to the material hardness $H$. That is

$\dot{w}=\frac{k_{\mathrm{w}}}{H} p_{3}\|\mathbf{S}\|$

The proportionality constant $k_{\mathrm{w}}$ is known as the wear coefficient and depends on the normal load and on the slip velocity. The bibliography contains the values of wear coefficients obtained experimentally as a function of these parameters [23]. In the present study it is assumed that the wear coefficient does not depend on the variations of the normal contact tractions or local slips within the contact patch.

From the results of the model proposed in Section 2, for each computed discrete time it is possible to obtain the wear rate in each element by means of Eq. (8). The wear rate $\dot{w}^{J}$ is evaluated through the Archard model in each element centre of coordinates $\left(x_{1}^{J}, x_{2}^{J}\right)$. The wear algorithm requires a set of points to be generated formed by the previous $\left(x_{1}^{J}, x_{2}^{J}\right)$ plus points located at the edge of the contact patch. The union by segments of the latter must contain the former.

Next, an enhanced solution of the wear rate field is estimated through a Delaunay triangulation from the previous set of points. In each triangular element an approximation is made of the wear rate field from the nodes $\left(x_{1}^{J}, x_{2}^{J}\right)$ by means of a linear interpolation of the nodal values $\dot{w}^{J}$.

The calculation of the worn material is performed at a number of discrete points on the railhead. Fig. 5 shows an example of this discretisation. If at a discrete time $i$ the $k$-th point of the rail is located within the contact patch, a wear rate value $\dot{w}_{i}^{k}$ can be estimated through a linear interpolation of the nodal values in the triangular element containing the rail point. The total wear depth value in the rail node $k$ is computed as

$W^{k}=\Delta t \sum_{i=1}^{M} \dot{w}_{i}^{k}$

where $M$ is the number of discrete times at which the wear rate is calculated. An improved solution of the wear depth calculation can be attained if additional solutions of the contact problem are generated by means of linear interpolation of the parameters $\left(x_{1}^{J}, x_{2}^{J}\right)$ and $\dot{w}^{J}$, corresponding to two consecutive discrete times.

\section{Non-stationary contact model}

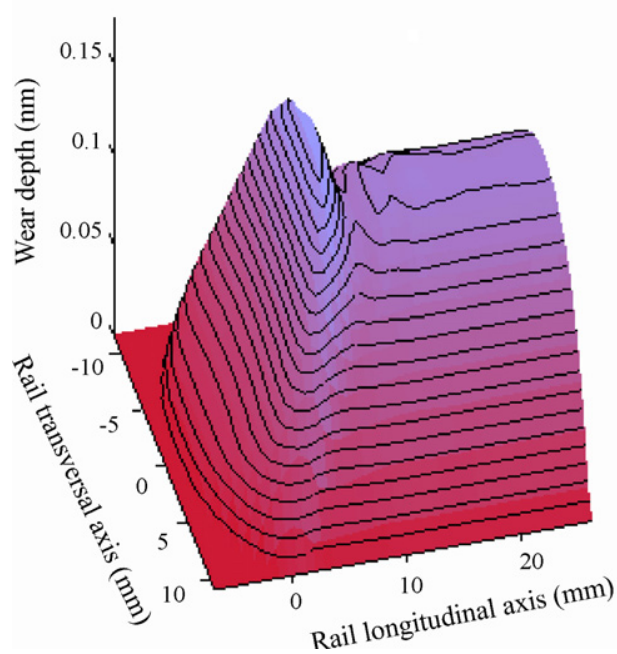

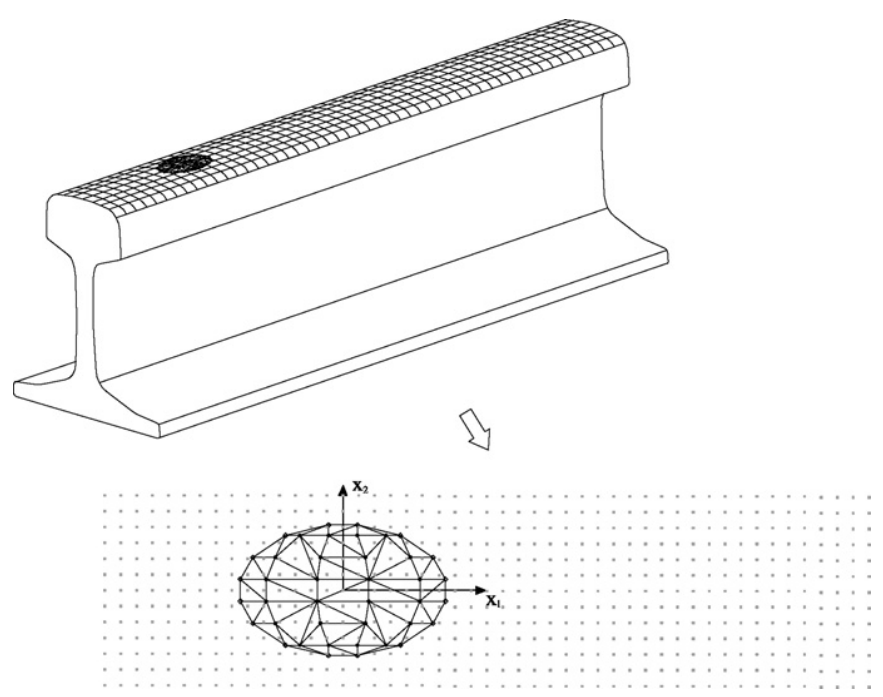

Fig. 5. Node generation on the railhead.

\section{Results}

The first calculation aims to establish the influence of the application of a contact model based on an Exact Theory. For this, a three-dimensional version of the well-known problem "From Cattaneo to Carter" is considered with the parameters contained in Table 1. From a practical point of view, this case could be compared to the sudden application of a traction torque in a driving wheel. The contact parameters obtained from the non-stationary model rapidly converge to their stationary values, resulting a deviation in relation to the creepage calculated by using FASTSIM of only $3 \%$. Fig. 6 shows the estimated wear calculated through both contact models. Although a significant difference can be appreciated in the zone where the contact area is located at the initial time, the wear shapes after this zone coincide. Nevertheless, a difference exists between the models of the order of $15 \%$, which can be attributed to the use of a Simplified Theory in FASTSIM.

In order to appreciate the effect of the rapid variation of the forces transmitted in the wheel-rail contact, two cases are considered

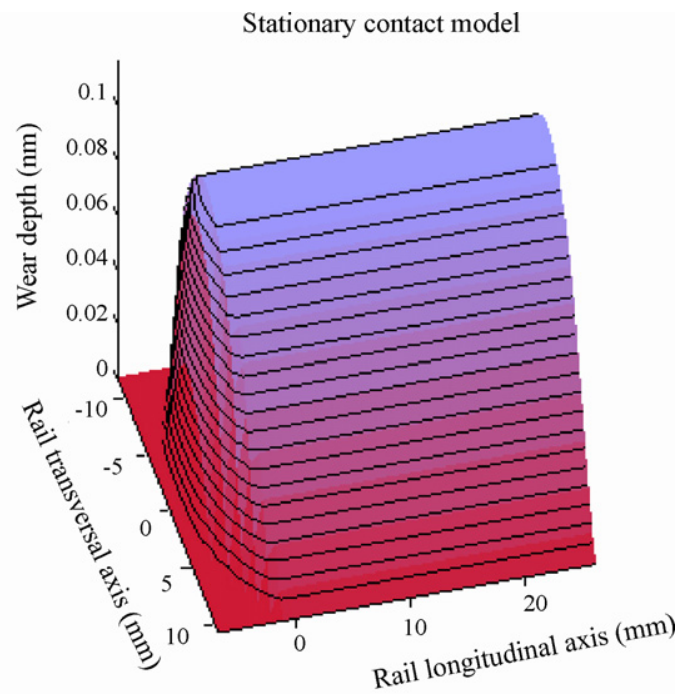

Fig. 6. Wear depth calculation when a constant longitudinal force is applied. 


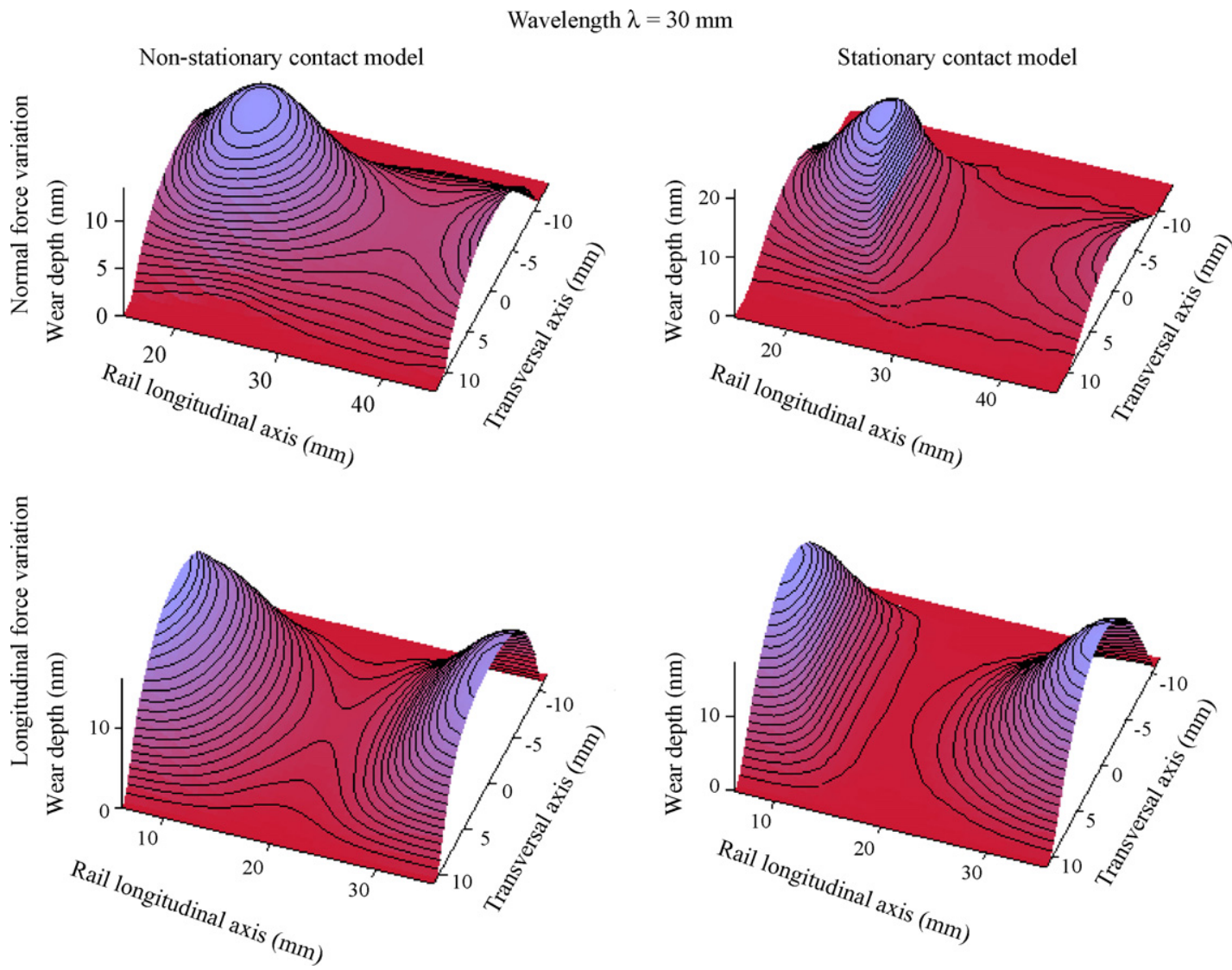

Fig. 7. Wear depth calculation when a $30 \mathrm{~mm}$ wavelength variable force is applied.

Wavelength $\lambda=15 \mathrm{~mm}$
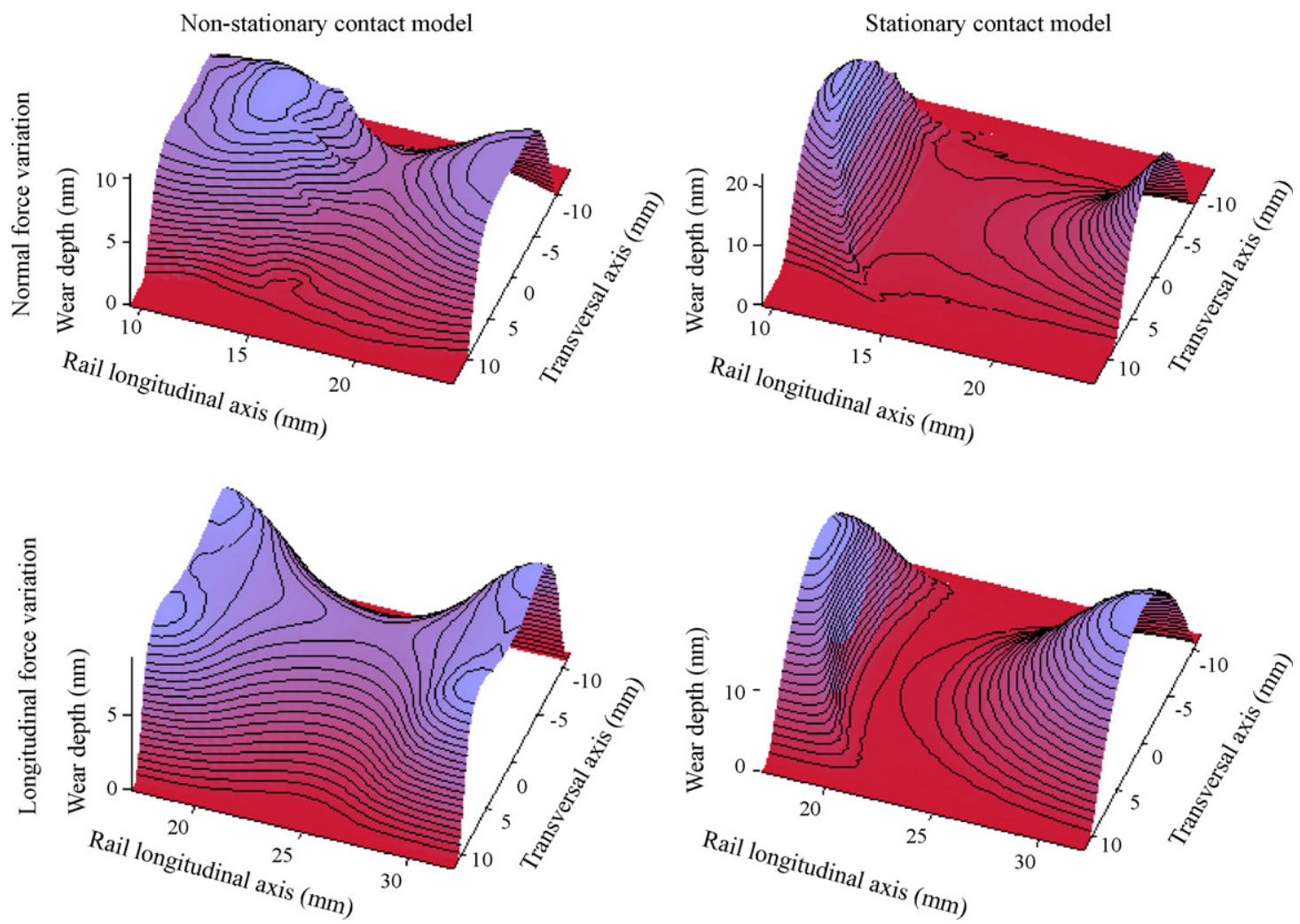

Fig. 8. Wear depth calculation when a $15 \mathrm{~mm}$ wavelength variable force is applied. 
- Variation of the normal force. This is the immediate consequence of a wheel rolling on a corrugated rail. A sinusoidal variation is analysed with different wavelengths $\lambda$. The force variation amplitude and the mean force are 45 and $100 \mathrm{kN}$, respectively.

- Variation of the longitudinal force. This case could be attributed to the torsional modes of the wheelset or to instabilities of the power train. In a similar way as the previous case, a sinusoidal variation of the longitudinal force is considered, where the force variation amplitude and the mean force are 10 and $20 \mathrm{kN}$, respectively.

In Figs. 7 and 8, the wear results derived from both contact models are presented. The rail stretch length analysed in these figures corresponds to that of a single cycle of the external force variation. In each figure, the upper graphics analyse the normal force variation while the lower ones result from the longitudinal force variation. The graphics shown on the left- and right-hand side are obtained through the non-stationary contact model and FASTSIM, respectively. The calculations in Fig. 7 correspond to a force variation associated with a wavelength of $\lambda=30 \mathrm{~mm}$, while those in Fig. 8 correspond to a wavelength of $15 \mathrm{~mm}$. Differences between results from both contact models are observed. These differences cannot be only attributed to the elastic model (Simplified or Exact Theory), but also to the contact process. These differences are more pronounced when the wavelength is reduced or the amplitude of the force variation increases.

\section{Conclusions}

In this paper, the influence of the non-stationary contact process on the estimation of corrugation patterns is investigated. To this end, a method implementing a wheel-rail contact model together with a methodology for computing the three-dimensional rail wear is developed.

The rail wear calculation method is based on the Archard's model. The main input parameter is the local slip provided by the contact model. An enhanced solution of the local slip field in the contact patch is obtained by combining a Delaunay triangulation and a linear interpolation among values at the vertices of each triangle. Consequently, smooth contact parameter fields are obtained. These results are assigned to the nodes of a regular mesh of the rail running surface.

The proposed non-stationary contact model is a modified version of CONTACT. In comparison with the original methodology based on the assumption of a potential contact area, this model uses a spatial discretisation adapted to the contact patch size. This assumption does not introduce errors when the normal force varies and therefore, it is adequate for simulating corrugation patterns on rails.

The results of the proposed method are compared with the results from analogous calculations in which FASTSIM is used for computing the contact parameters. With the aim of determining the influence of the elastic model independently of the non-stationary process, the conditions in which the contact process converges to its stationary values are simulated initially. In these calculations, differences of around 15\% between the wear parameters obtained through both methods are detected. FASTSIM is more accurate in relating creepages and external forces since it was adjusted through these parameters by means of the Linear Theory; however, the local slip field could be estimated less precisely. Taking into account the uncertainty inherent to the wear model and its parameters, the above mentioned differences may not be relevant as they do not affect to the general shape of the worn railhead.
The second calculation set considers those situations in which the external force (normal or longitudinal) vary rapidly. The faster the contact conditions vary, the more noticeable are the differences in the estimated wear depth field through both models. This fact implies that the type of contact model determines the corrugation pattern, mainly in the cases of short-wavelength defects. A tendency permitting the prediction of that deviation through the most simplified model cannot be appreciated.

The observed differences are mainly due to the non-stationary contact process. In comparison with a stationary model, the contact parameters computed by using the non-stationary contact model are out of phase with the externally applied force. In addition, reverse contact or slip areas in the leading edge of the contact patch may appear when employing a non-stationary model. These phenomena may determine the wear pattern of the rail running surface.

\section{Acknowledgements}

This research has been financed by the Projects T79/2006 (Ministerio de Fomento, Metro de Madrid, CDM) and TRA 2004 01828/TREN (Ministerio de Educación y Ciencia, FEDER, TALGO).

\section{Appendix A. Supplementary data}

Supplementary data associated with this article can be found, in the online version, at doi:10.1016/j.wear.2008.01.024.

\section{References}

[1] S.L. Grassie, J. Kalousek, Rail corrugation: characteristics, causes and treatments, Proc. Inst. Mech. Eng., Part F: J. Rail Rapid Transit 207 (1993) 57-68.

[2] J. Kalousek, S.L. Grassie, Rail corrugation: causes and cures, Int. Railway J. (July) (2000) 24-26.

[3] K. Hempelmann, F. Hiss, K. Knothe, B. Ripke, The formation of wear patterns on rail tread, Wear 144 (1991) 179-195.

[4] A. Igeland, H. Ilias, Rail head corruption growth predictions based on non-linear high frequency vehicle/track interaction, Wear 213 (1997) 90-97.

[5] C. Andersson, A. Johansson, Prediction of rail corrugation generated by threedimensional wheel-rail interaction, Wear 257 (2004) 423-434.

[6] A. Johansson, Out-of-Round Railway Wheels-Causes and Consequences, Thesis for the degree of Doctor of Philosophy, in: Department of Applied Mechanics, Chalmers University, Sweden, 2005.

[7] J.J. Kalker, Three-Dimensional Elastic Bodies in Rolling Contact, 1st ed., Kluwer, 1990.

[8] Z. Li, Wheel-Rail Rolling Contact and its Application to Wear Simulation, Thesis for the degree of Doctor of Philosophy, Delft University, Holland, 2002.

[9] X.S. Jin, Z.F. Wen, K.Y. Wang, Effect of track irregularities on initiation and evolution of track corrugation, J. Sound Vibration 285 (2005) 121-148.

[10] X.S. Jin, K.Y. Wang, Z.F. Wen, W.H. Zhang, Effect of rail corrugation on vertical dynamics of railway vehicle coupled with a track, Acta Mech. Sin. 21 (2005) 95-102.

[11] X.S. Jin, Z.F. Wen, K.Y. Wang, Z.R. Zhou, Q.Y. Liu, C.H. Li, Three-dimensional train-track model for study of rail corrugation, J. Sound Vibration 293 (2006) $830-855$.

[12] L. Baeza, A. Roda, J. Carballeira, E. Giner, Railway train-track dynamics for wheelflats with improved contact models, Nonlinear Dyn. 45 (2006) 385-397.

[13] L. Baeza, A. Roda, J.C.O. Nielsen, Railway vehicle/track interaction analysis using a modal substructuring approach, J. Sound Vibration 293 (2006) 112-124.

[14] A. Gross-Thebing, K. Knothe, K. Hempelmann, Wheel-rail contact mechanics for short wavelengths rail irregularities, Vehicle Syst. Dyn. 20 (1991) 210-224.

[15] A. Gross-Thebing, Frequency-dependent creep coefficients for threedimensional rolling contact problems, Vehicle Syst. Dyn. 18 (1989) 359374.

[16] K. Knothe, A. Gross-Thebing, Derivation of frequency dependent creep coefficients based on an elastic half-space model, Vehicle Syst. Dyn. 15 (1986) 133-153.

[17] K. Knothe, A. Gross-Thebing, High-frequency contact mechanics: the derivation of frequency-dependent creep coefficient, Proc. Inst. Mech. Eng., Part C: Mech. Eng. Sci. 198 (1984) 167-173.

[18] A. Gross-Thebing, Lineare Modellierung des instationären Rollkontaktes von Rad und Schiene, Thesis for the degree of Doctor of Philosophy, Technische Universität Berlin, Berlin, 1993.

[19] J.B. Nielsen, New Developments in the Theory of Wheel/Rail Contact Mechanics, Thesis for the degree of Doctor of Philosophy, Department of Mathematical Modelling, Technical University of Denmark, Denmark, 1998. 
[20] J.F. Archard, Contact and rubbing of flat surfaces, J. Appl. Phys. 24 (1953) 981-988.

[21] L. Baeza, F.J. Fuenmayor, J. Carballeira, A. Roda, Influence of the wheel-rail contact instationary process on contact parameters, J. Strain Anal. Eng. Des. 42 (2007) 377-387.
[22] B. Paul, J. Hashemi, Contact pressures in closely areas in the leading edge of the contact patch and/or conforming elastic bodies, J. Appl. Mech.-Trans. ASME 48 (1981) 543-548.

[23] T. Jendel, Prediction of wheel profile wear-comparisons with field measurements, Wear 253 (2002) 89-99. 